gelatinase expressed in three major forms: dimer, monomer and a complex with neutrophil gelatin-associated lipocalin (NGAL). Interleukin-(IL)-6 is a pleiotropic cytokine expressed by a variety of immune and non-immune cells. However, the mechanisms by which IL-6 contributes to the pathogenesis of chronic arthropathies are not fully understood.

Objectives: The purpose of the present work was to perform a comparative study of the IL-6 production and MMP-9 activity in FLS stimulated with SF from patients with osteoarthritis $(\mathrm{OA})$, rheumatoid arthritis (RA) or spondyloarthritis $(\mathrm{SpA})$. In addition, the effect of IL- 6 blockade on MMP-9 activity was evaluated.

Methods: Primary FLS were obtained from SF of the RA patients. Furthermore, the SW982 human synovial cell line was used. The SF of patients with OA $(n=11)$, RA $(n=11)$ or SpA $(n=9)$ patients were pooled. The FLS were stimulated with OA, RA or SpA SF pools and supernatants (SN) were collected after 24, 48 and 72 h. The IL-6 levels were assessed in the SN by ELISA. The gelatinase activity of the SN was determined by zymography. The IL-6 function was blocked with the anti-IL-6 receptor antagonist tocilizumab (TCZ) $(200 \mu \mathrm{g} / \mathrm{ml})$.

Results: Earlier induction of IL-6 in SW982 cell line was observed by RA and SpA SF stimulation since significant levels were detected at $24 \mathrm{~h} \quad(\mathrm{p}<0.001$ and $p<0.01$ compared with non-stimulated cells, respectively), whilst OA SF induced significant IL-6 secretion at $72 \mathrm{~h}(\mathrm{p}<0.01)$. Similar results were observed in primary FLS. In contrast to SF of OA patients, SF of patients with RA or $\mathrm{SpA}$ induced increased and sustained secretion of active MMP-9. Moreover, the molecular weight band corresponding with NGAL-MMP-9 complex, considered a protected form of MMP-9, was detected with higher intensity in the SN of FLS stimulated with RA or SpA SF compared with OA SF $(p<0.001)$. In the presence of TCZ, significant inhibition in the gelatinase activity of all MMP-9 forms was observed at $48 \mathrm{~h}$ of stimulation with RA or SpA SF $(\mathrm{p}<0.001$ for MMP-9 dimer and NGAL-MMP-9 complex; $p<0.01$ for MMP-9 monomer, compared with FLS stimulated in absence of TCZ).

Conclusions: We conclude that SF of patients with inflammatory arthritis recreate a differential microenvironment for FLS that impacts on early phenotypic changes of these cells. The IL-6 provokes augmented and persistent MMP-9 activity in FLS stimulated with RA or SpA SF. This work identifies TCZ as an inhibitor of all forms of MMP-9.

Disclosure of Interest: None declared

DOI: 10.1136/annrheumdis-2017-eular.2940

\section{AB0052 INCREASED PERIPHERAL CD8+ T CELL RESPONSES IN SLE BY LOW-DOSE IL-2 TREATMENT}

R. Zhang, J. He, X. Sun, Y. Zou, Y. Gan, Z. Li. Peking University People's Hospital, Beijing, China

Background: CD8+ $T$ cell responses to viral pathogens is crucial for the prompt resolution of acute infections. SLE patients are more likely to have infections due to suppression of immune system by long-term glucocorticoid and immunosuppressive agent intake. Our previous study showed that low-dose IL-2 is effective in SLE.

Objectives: The present study is to evaluate the potential anti-infection effect of low-dose IL-2 in refractory SLE patients.

Methods: Nine refractory SLE patients and 9 health controls ( $\mathrm{HCs}$ ) were recruited three cycles of 1 million IU recombinant human IL-2 (rhlL-2), administered subcutaneously every other day for 2 weeks, followed by a 2-week break. The disease activitise were evaluated by rheumatologist. The frequencies of $T$ cell subsets were assayed by flow cytometry. Virus-specific CD8 T cells responses were determined based on TNF-a, IFN-g and Grazmy B producing CD8 T cells upon CMV-EBV-Flu (CEF) viral peptide pool stimulation and subsequent intracellular staining.

Results: Most patients showed good clinical responses after three cycles of low-dose IL-2 treatment. Clinical improvement was observed in SIR-4 response, improved complement 3 and 4 serum level and decreased anti-ds-DNA serum level. Functional profiling of CD8 T cells in low-dose IL-2 treated patients revealed an increased in the frequencies of CEF viral peptide specific TNF- $\mathrm{a}^{+}$ and Grazmy-B ${ }^{+}$CD8 T cells. Moreover, low-dose IL-2 treated patients showed stronger antigen-specific response demonstrated by an increased stimulated/nonstimulate TNF-a-producing CD 8 T cells proliferation fold. Compared with HCs, SLE patients showed significantly lower frequencies of CEF specific Grazmy-B producing CD8 T cell, and treatment with low-dose IL-2 significantly increased the frequency of these Grazmy- $\mathrm{B}^{+}$CD8 T cells in SLE patients.

Conclusions: Low-dose IL-2 treatment was effective and safe in refractory SLE patients. Virus-specific antigen-specific CD8 T cell response could be enhanced upon this treatment which might be potentially valuable in anti-infection in SLE. References:

[1] Bilate AM, Lafaille JJ. Induced CD4+Foxp3+ regulatory T cells in immune tolerance. Annu Rev Immunol. 2012;30:733-58.

[2] Blattman JN, Grayson JM, Wherry EJ, Kaech SM, Smith KA, Ahmed R. Therapeutic use of IL-2 to enhance antiviral T-cell responses in vivo. Nat Med. 2003;9(5):540-7.

[3] Palucka K, Banchereau J. Diversity and collaboration for effective immunotherapy. Nat Med. 2016;22(12):1390-1.

Acknowledgements: We thank Pro. Xin Lin for valuable suggestions to this manuscript. The research in the laboratory was funded by grants from the NSFC (21010000237, NSFC by Pro. Jing He and Zhanguo Li.
Disclosure of Interest: None declared

DOI: 10.1136/annrheumdis-2017-eular.3196

\section{AB0053 MANGIFERIN MODULATES TNF-ALPHA AND MMP-9 SIGNALING}

S. Somani ${ }^{1,2}, \mathrm{~K}$. Modi ${ }^{3} .{ }^{1}$ Pharmacology, RK University, Rajkot; ${ }^{2}$ Pharmacology, Torrent Pharmaceuticals, Gandhinagar; ${ }^{3}$ Pharmacology, B K Mody Government Pharmacy College, Rajkot, India

Background: Rheumatic manifestation such as arthritis, spondylitis are the most common frequent extra-intestinal manifestations of inflammatory bowel disease (IBD), affecting 6 to $46 \%$ of patients. IBD is an umbrella term with two most prevalent entities namely Crohn's disease and Ulcerative colitis, defined as idiopathic chronic, relapsing and remitting inflammatory condition of intestinal tract. MMP-9, a matrixin is involved in the degradation of extracellular matrix promoting inflammation. Studies on MMP-9 knockout mice illustrated protection from collagen induced rheumatoid arthritis (RA) and Dextran sulphate sodium (DSS) induced colitis. Furthermore elevated MMP9 levels can be found in the synovial fluid of RA patients as well as IBD patients signifying that targeting MMP9 may have therapeutic importance. Mangiferin, an active component of Mango has demonstrated anti-inflammatory effects in the treatment of rheumatoid arthritis with low side-effects ${ }^{[1,2]}$; but its usefulness in chronic remission and relapse phases of human IBD i.e. chronic DSS model have not screened till date.

Objectives: We investigated the therapeutic potential of Mangiferin; in a clinical relevant chronic model of DSS induced colitis in mice.

Methods: Female BALB/c mice ( 8 to 12 wks) were randomized into four groups. Colitis was induced by cyclical administration of $5 \%$ DSS to mice i.e. 3 cycles of DSS with every cycle consisting of 7 days DSS followed by 7 days of autoclaved drinking water (7D DSS + 7D water). Group I (Normal control): free access to autoclaved drinking water. Group II (DSS control): free access to 5\% DSS. Group III (DSS + Mangiferin_30mg/kg): free access to $5 \%$ DSS + oral Mangiferin at $30 \mathrm{mg} / \mathrm{kg}$. Group IV (DSS + Mangiferin $60 \mathrm{mg} / \mathrm{kg})$ : free access to $5 \%$ DSS + oral Mangiferin at $60 \mathrm{mg} / \mathrm{kg}$. Mangiferin treatment was initiated following second cycle of DSS (i.e. Day 21); after assuring that colitis relapsed in mice. One fragment of the colon was fixed in $10 \%$ neutral buffered formalin for microscopic examination while the remaining tissue was divided into parts and stored at $-70^{\circ} \mathrm{C}$ for assessment of biochemical markers of oxidative stress and inflammatory cytokines such as TNF- $\alpha$, IL-1 $\beta$, MMP-9.

Results: Mangiferin treatment ameliorated the clinical parameters (body weight loss, stool consistency, occult blood), reduced microscopic damage (re-established mucosal architecture, abridged neutrophil infiltration), restored epithelial barrier integrity (diminished goblet cell loss), attenuated biochemical markers of oxidative stress (GSH, CAT, SOD, MDA, MPO), crucial inflammatory cytokines TNF- $\alpha$, IL-1 $\beta$ and attenuates MMP-9 levels implicated in the pathogenesis of arthritis and IBD. Conclusions: Considering the beneficial effects of Mangiferin in arthritis and IBD, we suggest that it would be valuable to use Mangiferin in IBD patients with arthritis as its extra-intestinal manifestation.

\section{References:}

[1] Luczkiewicz, P., et al., Mangiferin: A promising therapeutic agent for rheumatoid arthritis treatment. Med Hypotheses, 2014. 83(5): p. 570-4.

[2] Tsubaki, M., et al., Mangiferin suppresses ClA by suppressing the expression of TNF-alpha, IL-6, IL-1beta, and RANKL through inhibiting the activation of NF-kappaB and ERK1/2. Am J Transl Res, 2015. 7(8): p. 1371-81.

Disclosure of Interest: None declared

DOI: 10.1136/annrheumdis-2017-eular.4936

\section{AB0054 CXCL4 POTENTIATES TLR-DRIVEN POLARIZATION OF HUMAN DENDRITIC CELLS TOWARDS CYTOKINE PRODUCTION, ANTIGEN CROSS-PRESENTATION AND INCREASES STIMULATION OF CD8+T-CELLS}

S.C. Silva-Cardoso ${ }^{1,2}$, A.J. Affandi ${ }^{1,2}$, L. Spel ${ }^{2,3}$, M. Cossu ${ }^{1,2}$, M. Boes ${ }^{2,3}$, T.R. Radstake ${ }^{1,2} .{ }^{1}$ Depart. of Rheumatology \& Clinical Immunology; ${ }^{2}$ Laboratory of Translational Immunology; ${ }^{3}$ Department of Pediatrics, University Medical Center, Utrecht, Netherlands

Background: CXCL4 is a chemokine produced by activated platelets and immune cells. Several studies have reported that CXCL4 plays a critical role on physiological processes since it affects the proliferation and maturation of megakaryocytic and hematopoietic progenitor cells, regulates coagulation and wound healing, displays anti-tumoral and angiostatic activity and induces immune cell modulation. Dysregulation of these processes causes the disturbance of the immune system and homeostasis, and might lead to pathological conditions. Indeed, a strong correlation was previously found between elevated CXCL4 levels in the circulation and the clinical features of patients with systemic sclerosis (SSc) (1). Dendritic cells are essential players in innate defence and bridging towards adaptive immune responses, thereby contributing to both immune activation and maintenance of homeostasis.

Objectives: Considering previous observations on the association of dendritic cells and T-cell dysfunction in SSc, we here investigated the effect of CXCL4 on monocyte-derived DC (moDC) differentiation, on Toll-like receptor (TLR)-mediated responses and on activation of polyclonal and antigen-specific $C D 8^{+} T$-cells. 